\title{
Design and Implementation of the Automatic Assessment System for the Command and Control Specialty in Ships
}

\author{
Hao Zhang ${ }^{1,2, a}$, Zheng-yi Wang ${ }^{2}$ and Wei Yin ${ }^{1}$ \\ ${ }^{1}$ Dalian Naval Academy of the PLA, 1160018 Dalian, China \\ ${ }^{2}$ Naval Command College, 210016 Nanjing, China
}

\begin{abstract}
On the basis of analyzing the actual demand of automated assessment system for the command and control specialty in ships, the thought of the overall design of automated assessment system for the command and control specialty in ships is given, and the concrete realization methods of the user login module, test paper operation module and system maintenance module are studied and demonstrated. The proposed design idea and implementation method of automated assessment system for the command and control specialty in ships is scientific, efficient and practical, and provides reference for the exploitation of automated assessment system for the command and control specialty in ships.
\end{abstract}

\section{Introduction}

Currently, The construction of automated assessment system for the command and control specialty in ships lags. The automated assessment system used in troops have the shortcomings of poor objectivity of schema, low degree of intelligence, and poor completeness of functional. Thus it is difficult to meet the practical needs of the talent training and assessment of the forces in the new period [1-3]. In order to resolve the shortage of the current system and improve the assessment efficiency of professional ability of command and control specialty in troops, the high validity, strong extensibility and good open automated assessment system for the command and control specialty in ships is studied, designed and implemented by using the advanced theory and method of system engineering theory, object-oriented programming technology and relational database and so on.

\section{Demand Analysis}

There are two main tasks of the automated assessment system for the command and control specialty in ships, one is to provide more convenient means to collect the questions, and the other one is to organize, edit and print the test paper flexibility according to the requirement of the question and the user. In the process of management and use of the question bank system, the most important is the autonomy and flexibility of the question bank system, which can be embodied in the following three aspects.

\subsection{Independent initialization of the question bank}

a Corresponding author: zhanghao_zhh_81@126.com
Users have the right to create a new question bank. There are major defects of part of the question bank which selling on the market, such as not built a new question bank or the user has no right to modify the attribute of the test questions which stored in the question bank. This problems will restrict the authority of the system. The administrator would like to create a new question bank, or add and change the attributes and classification scheme according to demand.

\subsection{Independent maintenance of the question bank}

Users have the right to add, modify and delete the test questions. The test questions which sold in the market have no scalability and openness. Therefore, the users have no complete control over the question bank [4]. The question bank such as the above will not be able to keep up with the times and will be aging and losing vitality in a relatively short time. For the automation assessment system for the command and control specialty in ships, faculty members clearly want to have the right to control, including the entry, the deletion and the change of the test questions and so on.

\subsection{Flexibly call for the question bank}

Users will be able to extract the test questions from the question bank on their own. Although the question bank system on the market have this basic function, but the selectivity is not available for the user, so it can't meet the needs of specific specialty, and most of the system does not allow the modification of generated test paper and have poor compatibility. For the command and control 
specialty in ships, each assessment will have different emphases, so the assessment system must meet the assessment requirements.

\section{The overall design}

\subsection{Overall design}

According to the demand analysis of the automated assessment system for the command and control specialty in ships, the overall technology roadmap of system is shown in figure 1. The system has three modules: user login module, test paper operation module and system maintenance module.

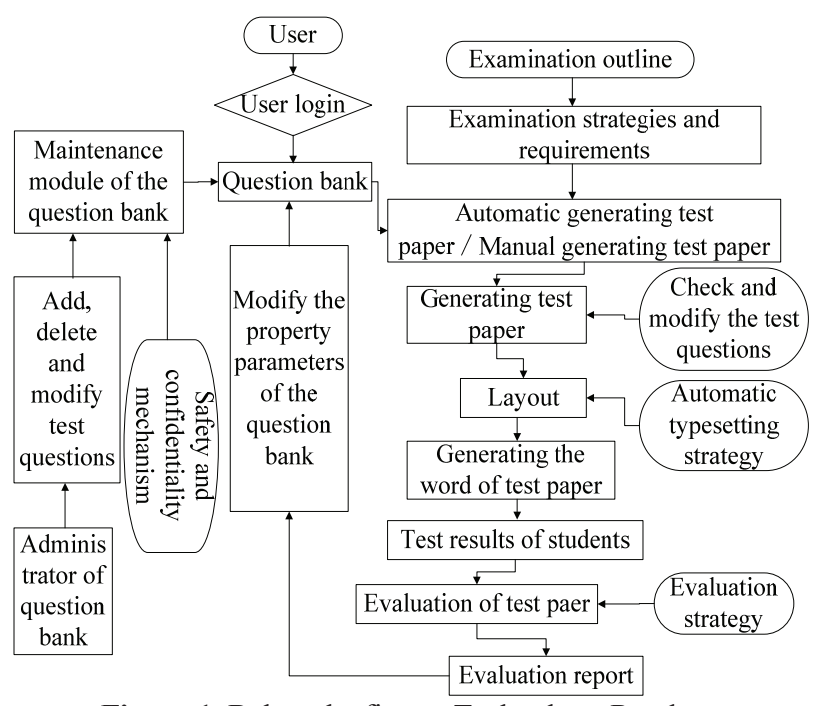

Figure 1. Below the figure. Technology Roadmap.

\subsection{User login module design}

The system can set different permissions for users of all access systems, and can divide users into three categories: administrators, experts and assessment objects. The first type is the administrator, who has the highest authority. The administrator not only has full access to the test module and system maintenance module, but also can read other users' information and modify the user's rights. The second category is experts, who have full access to test paper operating modules and system maintenance modules, but cannot access other user information. The third category is the assessment object, which can only browse the generated test paper or answer sheet when accessing the question bank system, but unable to carry out generating paper and operating on the test questions and the question bank.

\subsection{Design of test paper operation module}

The test paper operation module is divided into the generating paper sub-module and the paper analysis submodule.

\subsubsection{Generating paper}

Generating paper can be divided into manual generating paper and automatic generating paper. After the user enter to generating paper module, the user needs to input the requirements of generating paper according to the requirement of the exam outline and exam strategy, and system will form the constraint matrix on the basis of the above requirement. If the user selects the automatic generating paper, the system will generate the test paper by calling the random function method in the question bank. If the user selects the manual generating paper, the system will retrieve the test questions in the question bank for the user to select, and the system will automatically generate the test paper after the user has chosen to complete the test.

\subsubsection{Test paper analysis}

It consists of historical analysis and test questions feedback. After the completion of the examination, the faculty members can use the question bank system to analyze the test paper, the analysis results will be saved in the form of statistics of the question bank system. At the same time, the property of the test questions will be feedback to question bank in the form of a certain parameter.

\subsection{System maintenance module design}

The system maintenance module is divided into question bank operation sub-module and test questions operation sub-module. The question bank operation is the user who have the authority of administrators and teachers to design the storage scheme as well as attribute name and attribute values of test questions according to their own needs, so as to realize the personalized and flexibility design of question bank. The test questions operation is the user who have the authority of administrators and teachers to have right to operate and edit all the test questions in the question bank, so as to update the test questions in time.

\section{Implementation of functional module technology}

\subsection{Create application objects}

\subsubsection{Create workspace}

In PowerBuilder, click the New icon in the toolbar to pop the New dialog box. Select the Workspace tab in the dialog box, then select the Workspace tab and click the OK button. In the save dialog box that pops up, input the filename to save and select the saved path, and finally click the save button.

\subsubsection{Create new application objects}

Click the New icon in the toolbar, pop the New dialog box, select the Application item on the Target tab, and 
click the OK button. Input the name of the application object, the name of the application library, and the name of the target object in the pop-up dialog box, and click the Finish button.

\subsubsection{Connect to the database}

Write the following statement in the Open event of the current application:

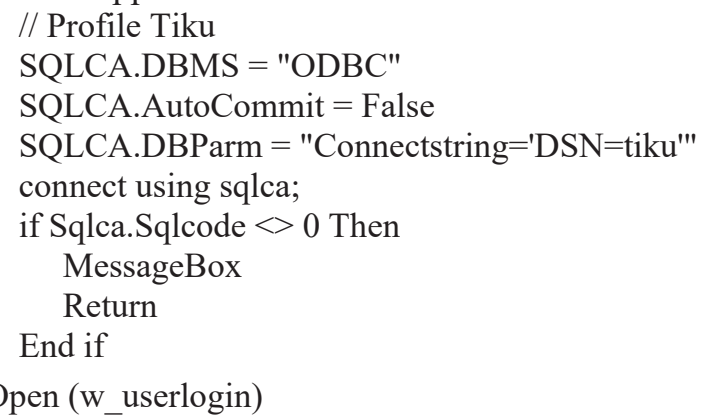

\subsection{Create a database}

Click the DB Profile icon in the toolbar, and select ODB ODBC $\rightarrow$ Utilities $\rightarrow$ Create ASA $\rightarrow$ Database in the pop-up dialog box. Double-click the selected item and pop up the dialog box. In this dialog, fill in the database information, including User ID, Password, Database Name and other attribute information.

Once the database have been created, and then the tables can be created. This database includes five kinds of tables: user information table, test questions type table, test paper table, examinee answer table, auxiliary coding table. There are two ways to create a table, one is that visually creates tables and the other one is that creates tables with SQL statements.

\subsection{Implementation of functional modules}

The required windows for the command and control system and combat use in ships are as follows. The creation methods and procedures of these windows are described in detail as follows.

\subsubsection{Create the main menu window}

The main menu window is the basis of many windows in the command and control system and combat use in ships, and some of the subsequent windows can be inherited from it. The function of the main menu window is to implement the main menu in the question bank system, which can enter the corresponding window by clicking the menu items in the main menu. In the PowerBuilder toolbar, click on the New icon and select the window item in the PB Object page. After creating the window, you can set properties window, such as the title of the window in the General page and settings window size in the Other page, etc. Finally, use the w_main name to store the window.

\subsubsection{Specific implementation of user login module}

(1) Create a user login window

The main function of the user login window is to determine whether the visitor is the user of the question bank of the command and control system and combat use in ships, and what privileges the user has. The Name of the first single line editor in the window pane is set as sle user, and the Name of the second single-row editor is set as sle_password. Write the following code in the Clicked event of the "user login" button:

string ls user,ls password

ls user $=$ trim(sle user.text)

select password

into :1s_password

from user1

where id $=:$ ls user;

ls password $=$ trim(ls password)

if sle_user.text=" or sle_password.text $=$ " then messagebox

return

end if

if trim(sle_password.text) $=1 \mathrm{~s} \_$password then open(w_main)

close(parent)

else

messagebox

end if

In the Clicked event of the "register" button, the following code is written:

open(w_newuser)

close(parent)

(2) Create a user registration window

The user registration window is set up for new users who are not authorized to visit the command and control system and combat use in ships for the first time. In the window, the new user need to fill out personal information and their required permissions, if registered the users of faculty, the right registration code should be needed to fill in a registration code, otherwise the registration failure. After the creation of the window, and then save the window with the name of w_newuser1, and w_newuser2. Create a function of wf_save (), which is used to save the User's User name, password, and other personal information in the User information table (User).

[5] the code is as follows:

if dw_1.update ()$=1$ then commit;

else

rollback;

return

end if // save data

if isnull (ib_emp_pic) false then

string ls code

1s_code $=$ dw_1.getitemstring $(1$, "em_code" $)$

updatelob employees set picture $=: i \bar{b} \_$emp_pic

where em code $=: 1$ s code;

if sqlca.sqlnrows $<=\overline{0}$ then messagebox return

end if

In user registration window 2, write the following code in the Clicked event of the cb_zhuce "register" button: 
wf_save ( )

messagebox

open(w_userlogin)

close(parent)

\subsubsection{Specific implementation of the test paper module}

Test paper operation module is the most important module in the command and control system and the operational use in ships, which contains four windows: automatic generating test paper window, manual generating test paper window, extraction test paper window and statistical analysis window.

(1) create automatic generating test paper window

The main function of automatic generating test window is to set up the test information according to the test requirement, and the system will automatically generate the test paper after given the attribute parameters.

(2) create manual generating test paper window

The main function of manual generating of test paper window is to browse the question bank according to the questions or chapters, and manually select the appropriate questions to form the test paper.

(3) create extract test paper window

The test paper generated by the automatic generating test paper window and manually generated test paper window will be stored in the test paper table of the database. The main functions of the extract test paper window is selecting the generated test paper in the database, and browse the test questions in the test paper.

(4) create statistical analysis window

The statistical analysis window is set up to analyze the situation of the examination after completing the examination. In the statistical analysis window, the OLE control is used to draw the statistical table and the chart. It is used to transmit a computer application to another application information by adopting remote procedure calls. The user can not only save an object by using OLE, but also can deal directly with the server application object.

\subsubsection{Implementation of system maintenance module}

The system maintenance module is the main ways for administrators and teachers to manage and update the question bank. The main functions include the entry and maintenance of the test questions. Because of the different of the contents and properties in each topic, each topic separately create up the input and maintenance window, the module contains eight windows: choice question entry window, fills-in-the-blanks entry window, true or false entry window, essay entry window, choice question maintenance window, fills-in-the-blanks maintenance window, true or false maintenance windows, essay maintenance window. This is to select the choice question entry window as an example to introduce its concrete implementation method.

In the choice question entry window, the user who have the authority of the administrator and faculty can input the questions and their attributes, including the question number, title, option, answer, difficulty and chapter. The code is written in the CloseQuery event of this window as follows [6] :

long ll_backvalue

if $\left(\overline{\mathrm{d}} \mathrm{w}_{-}\right.$1.modifiedcount ()$+\mathrm{dw} \mathrm{w}_{-}$1.deletedcount ()$\left.<>0\right)$ then

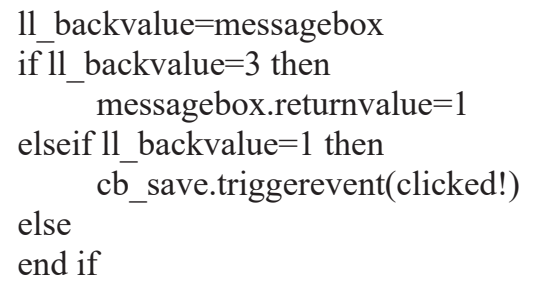

Create a function of wf_save (), which has no arguments or return values. The function of this function is to store the user's entry test questions and their property information into the database selection table (TopicSelect). The specific code is as follows:

if dw_1.updata( $)=1$ then commit;

else

rollback;

return

end if

long 11_row

11_row=w_newselect.dw_1.retrieve( )

string ls_code

ls_code $=\mathrm{dw}$ _l.getitemstring $(1$, "ts_code" $)$

long 11 findnum

11_findnum $=$ w_newselect.dw_1.find("ts_code $="$ "\& +1 ls code-+"'"',1,11_row)

w_newselect.dw_1.scrolltorow(11_findnum)

Write the following code in the "input" button's Clicked event:

wf_save()

dw_-1.reset()

dw_-insertrow $(0)$

dw_1.setfocus ()

\section{References}

1. Xiu Z H, Zhang H, Command and control system in ships and operational use (Tide Press, 2014)

2. Luo L, Liu Z, The key technologies of the future command and control system of the naval vessel, Modern Defense Technology, 31, 5 (2003)

3. Xu J, Xia H C, Match analysis model and simulation of shipborne $\mathrm{C} 2$ system and combat task, Command Control \& Simulation, 32, 5 (2010)

4. Jeffrey D, Ullman, Jennifer W, A first course in database systems (1999)

5. Li H J, Liu C R, Case development of PowerBuilder (Electronic Industry Press, 2009)

6. Yin H X, Zhang W M, PowerBuilder practical course (Electronic Industry Press, 2009) 